\title{
Design of Square Substrate Integrated Waveguide Cavity Resonators: Compensation of Modelling Errors by Support Vector Regression Machines
}

\author{
Giovanni Angiulli \\ Departement of DIMET, University of Mediterranea, Via Graziella n.189121 Reggio Calabria, Italy
}

Received 2012-08-29, Revised 2012-09-01; Accepted 2012-09-05

\begin{abstract}
The design of Substrate Integrated Waveguide (SIW) resonators is usually a cumbersome process, especially due to the length "trial and error" procedure involved in this task. In this study Support Vector Regression Machines (SVRMs) are employed to compensate the modeling errors associated to the design of SIW rectangular cavity resonators. To validate the proposed approach, we have compared the design outputs provided by our method with the results provided by commercial full wave software. The comparison between our predictions and the full wave simulations validate the effectiveness of the proposed approach.
\end{abstract}

Keywords: Resonators, Substrate Integrated Waveguides, Support Vector Regression Machines

\section{INTRODUCTION}

Substrate Integrated Waveguides (SIW) are devices built in a board of laminate by realizing arrays of metallic via holes to create a wave guiding channel or a cavity. They are an effective alternative to metallic waveguides mainly due to their simplicity and reduced costs of realization (Deslandes and $\mathrm{Wu}, 2001$ ). These motivations make SIW devices suitable for mass scale production. Several SIW devices are based on SIW resonators (Woo et al., 2007).

In particular, SIW resonators are dielectric structures loaded by metal layers on the top and the bottom and having walls realized by cylindrical vias of a given radius $\mathrm{a}_{0}$ arranged each other by a fixed distance $\mathrm{p}$, known as pitch (Fig. 1). An accurate design of these devices require a thorough understanding of the functional dependence of the operating resonant frequency from its geometrical and physical parameters. But such dependence is not known explicitly, even if dealing with standard-shaped structures. As a result, their design is usually cumbersome (Angiulli et al., 2009). Angiulli et al. (2009) a characterization of SIW resonator using a computational intelligence approach by means of Support Vector Regression Machines (SVRMs) has been given. Results shown in (Angiulli et al., 2009) demonstrated that the SVRM models provide a meaningful reduction of the overall computational burden due to numerical simulation of these devices associated with an accurate capability of modelling their behavior.

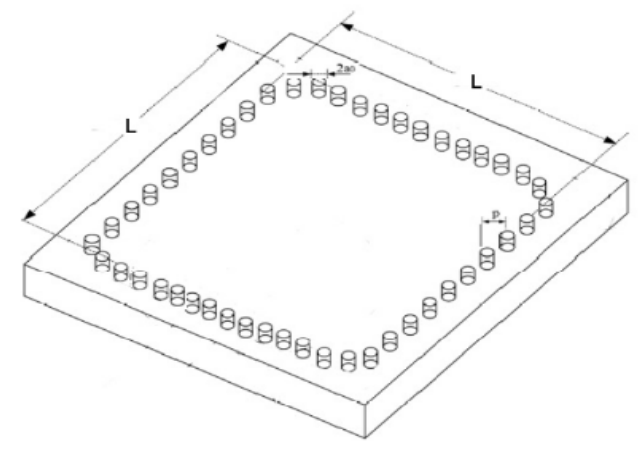

Fig. 1. A square SIW cavity resonator

In this study, following the approach proposed in (Carlo and Tringali, 2010) in the case of circular SIW resonators, empirical design formulas for a square SIW cavity resonator are combined in a loop of feedback with a SVRM trained to simulate the behavior of this device in a way to compensate errors due to model approximations. The study is organized as follows: we started introducing the technique of evaluation of resonances in a SIW resonator, recalled the basics on SVRM, then discussed our compensation procedure. Finally numerical results and conclusions are given.

\section{MATERIALS AND METHODS}

\subsection{Evaluation of SIW Resonances}


The analysis of a SIW waveguide through the magnetic dyadic Green's function has been presented in (Arnieri and Amendola, 2008; Amendola et al., 2008). The application of the boundary conditions involves two systems of algebraic linear equations, one for the TM scattering and the other for the TE scattering, that can be expressed in a matrix form as Equation 1:

$$
[\mathrm{L}]^{\mathrm{TE}, \mathrm{TM}}[\mathrm{A}]^{\mathrm{TE}, \mathrm{TM}}=[\Gamma]^{\mathrm{TE}, \mathrm{TM}}
$$

Theoretically SIW resonances are the complex frequencies $\mathrm{f}$ for which Equation 2 (Angiulli, 2007; Angiulli et al., 2007; 2009):

$$
\operatorname{det}\left([\mathrm{L}(\mathrm{f})]^{\mathrm{TE}, \mathrm{TM}}\right)=0
$$

However, from a practical point of view, the singularity of the matrix operator $[\mathrm{L}]^{\mathrm{TE}, \mathrm{TM}}$ can be efficiently tested only by means of the Singular Value Decomposition (SVD) (Golub and Loan, 1996). To explain this point, first of all we recall that the smallest singular value $\sigma_{\mathrm{n}}$ of $[\mathrm{L}]^{\mathrm{TE}, \mathrm{TM}}$ represents the actual distance between this matrix operator and the set of all matricial operators whose rank is less than or equal to $n$ 1. Hence, provided a threshold $\varepsilon>0$, we state that $[\mathrm{L}]^{\mathrm{TE}, \mathrm{TM}}$ is $\varepsilon$-singular, whenever $\sigma_{\mathrm{n}} \leq \varepsilon$. Starting from this condition, the set of the complex resonant frequencies $f$ $=\mathrm{f}_{\mathrm{re}}+\mathrm{j} \mathrm{f}_{\mathrm{im}}$ for which the matrix operator $[\mathrm{L}]^{\mathrm{T}, \mathrm{TM}}$ is $\varepsilon^{-}$ singular can be evaluated seeking for the minima of the two variable function $\sigma_{\mathrm{n}}\left(\mathrm{f}_{\mathrm{re}}, \mathrm{f}_{\mathrm{im}}\right)$ in an assigned band $\left[f_{\min }, f_{\max }\right]$ of frequencies. Angiulli et al. (2009) an effective search strategy is discussed, essentially consisting of an approximate computation of $\sigma_{\mathrm{n}}$ rather than a direct complete SVD factorization. First $\sigma_{n}\left(f_{r e}\right.$, $\mathrm{f}_{\mathrm{im}}$ ) is restricted to a real axis for locating its minima. Then these are used as starting points of a Muller routine (Antia, 2002) for a spread seeking through the whole complex plane.

\subsection{Support Vector Regression Machines}

The Support Vector Machines (SVMs) are heuristic structures able to solve classification problems (Scholkopf et al., 1999). SVMs can also be employed to solve regression problems, which is this case they specialize as Support Vector Regression Machines (SVRMs). In what follows we just recall the main theoretical issues on this topic, referring to (Scholkopf et al., 1999; Martinez-Ramon and Christodoulou, 2006) for more details. In order to explain the mathematical framework in which SVRMs are defined, let us consider the problem to approximate the set of input-output training data Equation 3:

$$
\left(x_{i}, y_{i}\right) \in R^{n} \times R, i=1, \ldots, 1
$$

By the so called support vector regression estimation function (Scholkopf et al., 1999; Martinez-Ramon and Christodoulou, 2006):

$$
\mathrm{f}(\mathrm{x})=\mathrm{w} \Phi(\mathrm{x})+\mathrm{b}
$$

In Equation $4 \mathrm{w}$ is a fixed vector of weights, $\mathrm{b}$ is a real number and $\Phi$ denotes a nonlinear mapping from $\mathrm{R}^{\mathrm{n}}$ to a high dimensional space, called feature space. The goal is to search for the value of the parameters $w$ and $b$ that minimize the regression risk Equation 5 (Scholkopf et al., 1999; Martinez-Ramon and Christodoulou, 2006):

$$
\mathrm{R}(\mathrm{f})=\frac{1}{2}\|\mathrm{~W}\|^{2}+\mathrm{C} \sum_{\mathrm{i}=1}^{\mathrm{m}} \Gamma\left(\mathrm{f}\left(\mathrm{x}_{\mathrm{i}}\right)-\mathrm{y}_{\mathrm{i}}\right)
$$

where, $\Gamma$ is the so-called $\varepsilon$-insensitive loss function (Scholkopf et al., 1999; Martinez-Ramon and Christodoulou, 2006):

$$
\Gamma\left(f\left(x_{i}\right)-y_{i}\right)=\left\{\begin{array}{cc}
0 & \text { if }|f(x)-y|<\varepsilon \\
|f(x)-y|-\varepsilon & \text { otherwise }
\end{array}\right.
$$

And $\mathrm{C}$ is a suitable constant that determines penalties to estimation errors. Higher penalties to errors are assigned by a large value of C so that SVM is trained to minimize error loosing in generalization ability, while a small value of this parameter allows a far higher generalization ability giving fewer penalties to error. The approximation for nonlinear data set is carried out with the use of a suitable kernel function, which implicitly defines the structure of the high dimensional feature space where a maximal margin hyperplane is found (Scholkopf et al., 1999; Martinez-Ramon and Christodoulou, 2006).

\subsection{The Compensation Procedure}

In Fig. 1 is shown a typical square SIW cavity resonator. The dominant resonant mode in this structure is the TE101. The resonant frequency is evaluated modelling this device as a conventional metallic cavity resonator (Woo et al., 2007):

$$
\mathrm{f}_{101}=\frac{\mathrm{c}}{\sqrt{\varepsilon_{\mathrm{r}}}}\left(\frac{\sqrt{2}}{2 \mathrm{~L}_{\text {eff }}}\right)
$$

where, $\mathrm{L}_{\text {eff }}$ is the effective length side of the SIW cavity, $\varepsilon_{\mathrm{r}}$ is the relative dielectric permittivity of the dielectric substrate and $c$ is the velocity of the light in vacuum. In Equation $6 \mathrm{~L}_{\text {eff }}$ is related to the radius $\mathrm{a}_{0}$ of the metallic vias and to the value of the pitch $\mathrm{p}$ by the following empirical relations (Woo et al., 2007): 


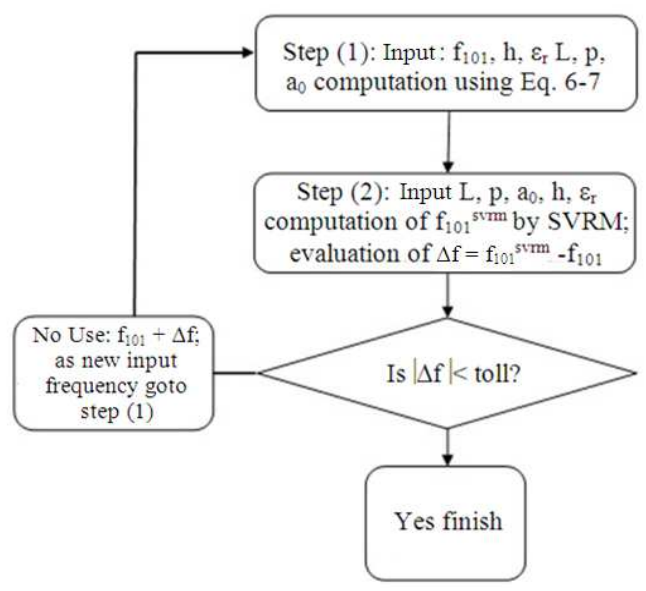

Fig. 2. Flow diagram of the proposed compensation procedure

$\mathrm{L}_{\text {eff }}=\mathrm{L}-\frac{\left(2 \mathrm{a}_{0}\right)^{2}}{0.95 \mathrm{p}}, \mathrm{p}<\frac{\lambda_{0} \sqrt{\varepsilon_{\mathrm{r}}}}{2}, \mathrm{p}<4 \mathrm{a}_{0}$

The Equation 6-7 allow to determinate the values of the geometrical parameters $\mathrm{a}_{0}, \mathrm{p}$ and $\mathrm{L}$ so that the square SIW resonator works at an assigned resonant frequency $\mathrm{f}_{101}$. But this design task, due to the empirical nature of the above relations, is fulfilled only in an approximate way so that a length "trial and error" procedure is required to tune the device. To overcome this problem we have exploited a computational intelligence approach using SVRMs. In particular starting from the values of L, $\mathrm{p}$ and $\mathrm{d}$, provided by Equation 6-7 a SVRM trained to model square SIW resonators, has been fed with these geometrical parameters in order to calculate the "actual" resonance frequency $\mathrm{f}_{101}^{\mathrm{svm}}$ of the structure at hand, possibly different from the design frequency $f_{101}$. Afterwards, a comparison between these frequencies is done and the design procedure is iterated in automatic way through a loop of feedback, until the value of the relative error Equation 8:

$\Delta f=\frac{\left|f_{101}-f_{101}^{s v m}\right|}{f_{101}}$

Is reduced under a given threshold, selected by the user, according to the flux diagram shown in Fig. 2.

\section{RESULTS AND DISCUSSION}

A SVRM capable to model square SIW resonators, exploiting a two degree polynomial $\operatorname{kernel}(\mathrm{C}=10, \varepsilon$ $=0.01)$ was implemented following the guidelines given in (Angiulli et al., 2008).
Table 1. Ranges of the considered input for the SVRM model exploited in this study (all dimensions are in millimeters, the ranges of $\mathrm{p}$ and $\mathrm{a}_{0}$ are evaluated by Equation 7 and not reported here)

\begin{tabular}{lll}
\hline $\mathrm{L}$ & $\mathrm{h}$ & $\mathrm{er}$ \\
\hline $2.0 \div 15.0$ & $0.318 \div 0.50$ & $2.5 \div 10.0$ \\
\hline
\end{tabular}

Table 2: Square Siw resonator designed using the compensation. $\left({ }^{*} \varepsilon_{\mathrm{r}}=3.35,{ }^{* *} \varepsilon_{\mathrm{r}}=2.55\right)$

\begin{tabular}{lllll}
\hline Project $\mathrm{n}$, & $\mathrm{f}_{101}(\mathrm{GHz})$ & $\mathrm{L}(\mathrm{mm})$ & $\mathrm{a}_{0}(\mathrm{~mm})$ & $\mathrm{p}(\mathrm{mm})$ \\
\hline $1^{*}$ & 10.00 & 11.897 & 0.337 & 1.350 \\
$2^{*}$ & 11.25 & 10.342 & 0.293 & 1.173 \\
$3^{*}$ & 9.75 & 12.198 & 0.346 & 1.384 \\
$4^{* *}$ & 11.22 & 12.640 & 0.368 & 1.227 \\
$5^{* *}$ & 12.10 & 11.365 & 0.281 & 1.125 \\
\hline
\end{tabular}

Table 3: Maximum absolute error $\delta$ err\%

\begin{tabular}{lllr}
\multicolumn{5}{l}{ Table 3: Maximum absolute error $\delta$ err\% } \\
Project $n$, & $\begin{array}{l}\mathrm{f}_{101} \text { by our compens } \\
\text { ation procedure }(\mathrm{GHz} 0\end{array}$ & $\begin{array}{l}\mathrm{f}_{101} \text { by HFSS } \\
\text { code }(\mathrm{GHz})\end{array}$ & $\delta$ err\%. \\
\hline 1 & 10.00 & 10.13 & 1.3 \\
2 & 11.25 & 11.42 & 1.5 \\
3 & 9.75 & 9.87 & 1.2 \\
4 & 11.22 & 11.32 & 0.9 \\
5 & 12.10 & 12.34 & 2.0 \\
\hline
\end{tabular}

The inputs of this machine were the dielectric permittivity of the dielectric substrate $\varepsilon_{\mathrm{r}}$, its thickness $t$, the pitch $\mathrm{p}$, the via hole radius $\mathrm{a}_{0}$ and the side length $\mathrm{L}$, while its output was the dominant mode resonant frequency $\mathrm{f}_{101}^{\text {svm }}$. In Table $\mathbf{1}$ are shown the ranges of variation of the inputs parameters. A training data set was obtained by numerical simulations using a Matlab code based on the computational technique for the evaluation of the SIW resonances discussed in the section "Materials and Method". Afterwards, different square resonators have been designed using a Matlab code implementing our compensation procedure (Table 2). Each project thus obtained has been checked re-computing its resonant frequency using the commercial code HFSS.

In Table $\mathbf{3}$ are shown the comparison between the full wave simulations and our predictions. The maximum absolute error observed is about $2 \%$, so suggesting the effectiveness of the proposed approach. Finally, it is notable that, once the SVRM has been trained (Angiulli et al., 2008), the compensation step is almost immediate. This makes the approach especially suitable for iterated applications.

\section{CONCLUSION}

In this study, a procedure to compensate the modeling error in the design of square SIW cavity 
resonator by using the SVRM approach, is given. Results have been compared with those obtained by full wave simulations carried out by the commercial software HFSS. The maximum absolute error obtained is about $2 \%$, confirming the effectiveness of the proposed approach.

\section{REFERENCES}

Amendola, G., G. Angiulli, E. Arnieri and L. Boccia, 2008. Resonant frequencies of circular substrate integrated resonators. IEEE Microw. Wireless Compon. Lett., 18: 239-241. DOI: 10.1109/LMWC.2008.918872

Angiulli, G., 2007. On the computation of nonlinear eigenvalues in electromagnetic problems. J. Electromagn. Waves Appli., 21: 527-532. DOI: 10.1163/156939307780616838

Angiulli, G., D.D. Carlo, G. Amendola, E. Arnieri and S. Costanzo, 2008. Support vector regression machines to evaluate resonant frequency of elliptic substrate integrate waveguide resonators. Progress Electromagn. Res., 83: 107-118. DOI: 10.2528/PIER08041803

Angiulli, G., E. Arnieri, D.D. Carlo and G. Amendola, 2009. Fast nonlinear eigenvalues analysis of arbitrarily shaped Substrate Integrated Waveguide (SIW) resonators. IEEE Trans. Mag.., 45: 14121415. DOI: 10.1109/TMAG.2009.2012650

Angiulli, G., M. Cacciola, M. Versaci, 2007. Microwave devices and antennas modelling by support vector regression machines. IEEE Trans. Mag., 43: 15891592. DOI: 10.1109/TMAG.2007.892480

Antia, H.M., 2002. Numerical Methods for Scientists and Engineers, 2nd Edn., Springer, ISBN: 3764367156, pp: 842 .
Arnieri, E. and G. Amendola, 2008. Analysis of substrate integrated waveguide structures based on the parallel-plate waveguide green's function, IEEE Trans. Microw. Theory Technol., 56: 16151623. DOI: 10.1109/TMTT.2008.925240

Carlo, D.D. and S. Tringali, 2010. Automatic design of circular SIW resonators by a hybrid aproach based on polynomial fitting and SVRM. J. Electromagn. Waves Appli., 24: 735-744.

Deslandes, D. and $\mathrm{K}$. Wu, 2001. Integrated microstrip and rectangular waveguide in planar form. IEEE Microw. Wireless Compon. Lett., 11: 68-70. DOI: 10.1109/7260.914305

Golub, G.H., C.F.V. Loan, 1996. Matrix Computations, 3rd, Annotated, Johns Hopkins University Press, ISBN-10: 0801854148, pp: 728.

Martinez-Ramon, M. and C.G. Christodoulou, 2006. Support Vector Machines for Antenna Array Processing And Electromagnetics. Morgan and Claypool Publishers. ISBN-10: 159829024X.

Scholkopf, B., C. Burges and A.J. Smola, 1999. Advances in Kernel Methods: Support Vector Learning. 1st Ed., MIT Press, ISBN-10: 0262194163, pp: 386.

Woo, L.I., S.H. Han, T.S. Yun, H. Nam and S.Y. Oh et al., 2007. A new Substrate Integrated Waveguide (SIW) cavity resonator with reflective characteristic. Proceedings of Asia Pacific Microwave Conference, Dec. 11-14, IEEE Xplore Press, Bangkok, pp: 1-4. DOI: 10.1109/APMC.2007.4554802 